\title{
Relationship between Aortic Arch Calcification, Detected by Chest X-Ray, and Renal Resistive Index in Patients with Hypertension
}

\author{
Adem Adar Orhan Onalan $^{a} \quad$ Hakan Keles $^{b}$ Fahri Cakan ${ }^{a}$ Ugur Kokturk $^{a}$

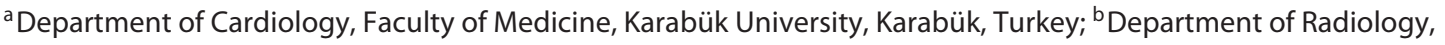 \\ Faculty of Medicine, Karabük University, Karabük, Turkey
}

\section{Significance of the Study}

- The renal resistive index (RRI) reflects intrarenal perfusion and is an important indicator of systemic hemodynamic changes. There is a need for predictors of increased RRI in hypertensive patients. Aortic arch calcification (AAC) is a direct indicator of arterial stiffness and hypertensive vascular damage and is evaluated by chest X-ray. AAC may thus prove to be a suitable predictor of RRI.

\section{Keywords}

Hypertension · Aortic arch calcification · Renal resistive index

\begin{abstract}
Objective: Aortic arch calcification (AAC) is a surrogate marker for arterial stiffness and hypertension-related vascular damage. Renal resistive index (RRI), a renal Doppler ultrasonography parameter, is used to assess renal hemodynamics. In this study, we aimed to evaluate the relationship between $\mathrm{RRI}$ and AAC in patients with hypertension. Methods: Patients with hypertension underwent a chest X-ray and renal Doppler ultrasonography. They were divided into two groups according to RRI (group 1: RRI $\geq 0.70$; group 2: RRI $<0.70$ ). Two examiners, blinded to the findings of RRI, reviewed the AAC in these patients. The kappa value was detected to be 0.781 and a $p$ value $<0.001$ was considered significant. Results: The study included 289 hypertensive patients (mean age $63.87 \pm 11.38$ years). $\ln 53.6 \%(n=155)$ of
\end{abstract}

the study subjects, RRI was observed to be $\geq 0.70$. Patients with $\mathrm{RRI} \geq 70$ were older and had more prevalent $A A C$ as well as left ventricular hypertrophy. A multiple linear regression analysis was carried out to test whether presence of AAC significantly predicted RRI. The results of the regression analysis indicated that presence of AAC significantly predicted RRI $(\beta=0.053 ; p<0.001)$. Conclusions: A strong and independent relationship was found between AAC on chest X-ray and RRI in patients with hypertension.

(c) 2018 The Author(s)
Published by S. Karger AG, Basel

\section{Introduction}

Hypertension is one of the most common cardiovascular risk factors. The diagnosis and treatment of hypertension-related end-organ damage together with regulation of blood pressure can help decrease cardiovascular mortality [1]. While the kidneys regulate blood pressure, they are also affected by any impairment in regulation of

\begin{tabular}{ll}
\hline KARGER & $\begin{array}{l}\text { (c) } 2018 \text { The Author(s) } \\
\text { Published by S. Karger AG, Basel }\end{array}$ \\
E-Mail karger@karger.com & $\begin{array}{l}\text { This is an Open Access article licensed under the Creative Commons } \\
\text { Attribution-NonCommercial-4.0 International License (CC BY-NC) } \\
\text { (http://www.karger.com/Services/OpenAccessLicense), applicable to } \\
\text { the online version of the article only. Usage and distribution for com- } \\
\text { mercial purposes requires written permission. }\end{array}$
\end{tabular}

Adem Adar

Department of Cardiology, Faculty of Medicine, Karabük University Sirinevler Mahallesi Alparslan Caddesi No:1

TR-78200 Karabük (Turkey)

E-Mail dradaradem@gmail.com 
blood pressure. Hypertension-related nephropathy is caused by a progressive loss of nephrons due to impaired regulation of blood pressure. Hypertensive nephropathy increases cardiovascular mortality, further impairing blood pressure regulation [2]. In clinical practice, one of the most accurate indicators of hypertensive nephropathy as detected by glomerular filtration rate (GFR) and microalbuminuria is renal resistive index (RRI) [3]. RRI is a calculation employed for evaluating various clinical conditions such as chronic renal allograft rejection, detection and treatment of renal artery stenosis, progression of chronic renal disease, and acute and chronic obstructive renal disease, and at the same time it reflects intrarenal perfusion changes as well [4]. RRI reflects intrarenal perfusion and is an important indicator of systemic hemodynamic changes. Therefore, it has a prognostic value in hypertensive patients [5]. Predictors of increased RRI are needed in hypertensive patients to find out the degree of renal impairment.

Aortic arch calcification (AAC) is caused by the progression of endothelial damage as well as inflammation and calcification in the aortic arch resulting from high blood pressure [6, 7]. Just like RRI, AAC is an important indicator of increased blood pressure burden and increased arterial stiffness $[8,9]$. AAC can easily be detected during routine chest radiography, which is a simple, inexpensive, and a widely available tool. Currently, no reports are available on the use of AAC for the prediction of RRI. The present study was therefore designed to investigate the relationship between AAC and RRI.

\section{Methods}

Patients with hypertension who reported to the Karabük University Hospital between January 2016 and January 2017 underwent a standard chest X-ray and renal Doppler ultrasonography. Two other physicians, who were not informed about the results of RRI, evaluated AAC using chest X-ray. The patients were divided into two groups according to RRI. Group 1 comprised patients with RRI $\geq 0.70$ and group 2 patients with $R R I<0.70$. Patients with a history of cardiac surgery, moderate to severe valvular heart disease, atrial fibrillation, renal artery stenosis, nephrostomy, malignancy, acute/chronic infective and inflammatory disease, and pregnant patients were excluded from the study. The following formula was used for calculating the sample size:

$$
\text { sample size }=\frac{Z^{2} \times(p) \times(1-p)}{d^{2}}
$$

where $Z=$ standard normal variate at $5 \%$ type 1 error $(p<0.05 ; 1.96$ was used in the formula), $p=$ expected proportion in a population based on previous studies or pilot studies, and $d=$ absolute error or precision (must be decided by the researcher).
According to the study by Berni et al. [10], the proportion of patients having RRI $\geq 0.70$ was found to be about $25 \%$. $<$ precision/ absolute error was kept at 5\% and type 1 error was kept at $5 \%$. Accordingly, applying these values in the formula above:

$$
\text { samplesize }=\frac{1.96^{2} \times(0.25) \times(1-0.25)}{0.05^{2}}=288 .
$$

All patients were examined for cardiovascular risk factors. Patients who had previously received oral antidiabetic and/or insulin therapy or those in whom fasting blood glucose was $\geq 126 \mathrm{mg} / \mathrm{dL}$ on at least two occasions were considered diabetic. Patients who had previously received antihypertensive treatment or who had blood pressure $\geq 130 / 80 \mathrm{~mm} \mathrm{Hg}$ on at least two occasions were identified as hypertensive. Pulse pressure was calculated as the difference between peak systolic and diastolic blood pressure. Patients with total cholesterol $>200 \mathrm{mg} / \mathrm{dL}$ or low-density lipoprotein cholesterol $>100 \mathrm{mg} / \mathrm{dL}$ or those who had received lipid-lowering medications in the past were considered to be hyperlipidemic [11]. Chronic kidney disease $(\mathrm{CKD})$ was defined as either kidney damage or a decreased GFR of $<60 \mathrm{~mL} / \mathrm{min} / 1.73 \mathrm{~m}^{2}$ for at least 3 months.

Albuminuria as a marker of kidney damage was defined as a urinary albumin excretion rate $\geq 30 \mathrm{mg} / 24 \mathrm{~h}$ [12]. We calculated the estimated GFR using the Chronic Kidney Disease Epidemiology Collaboration equation [13]. Active smokers or those who had quit smoking within the last 1 month were considered to be smokers. Body mass index was calculated by dividing the weight $(\mathrm{kg})$ by the square of height $\left(\mathrm{m}^{2}\right)$ [14]. Body surface area was calculated as the square root of the product of the weight $(\mathrm{kg})$ and height $(\mathrm{cm})$ divided by 3,600 [15]. All patients underwent 12-lead electrocardiography and transthoracic echocardiography. RRI was measured using renal Doppler ultrasonography by a radiologist who was blinded to patient information. Routine biochemical tests were performed in the patients after $12 \mathrm{~h}$ of fasting.

\section{Evaluation of $A A C$}

A standard (posterior-anterior) chest radiograph $(40 \times 40 \mathrm{~cm}$; Curix HT 1.000G Plus, Agfa, Mortsel, Belgium) was acquired with the patients standing up (Thoramat, Siemens, Erlangen, Germany). The focus-patient distance was set at $150 \mathrm{~cm}$. An automated exposure control having a fixed tube voltage of $117 \mathrm{kV}$ was used. AAC was graded as follows: grade 0 , no visible calcification; grade I, small spots of calcification or thin calcification on the aortic knob; grade II, one or more areas of thickened calcification; and grade III, circular calcification on the aortic knob [11] (Fig. 1). Two examiners, who were blinded to the findings of RRI, reviewed AAC on the chest radiograph. One hundred chest radiographs, randomly selected for the evaluation of AAC, were assessed independently by two cardiologists to assess the reliability of AAC diagnosis. The kappa value was detected to be 0.781 and $p$ to be $<0.001$.

\section{Measurement of RRI}

All examinations were performed by a single radiologist using the same device $(50-\mathrm{Hz}$ wall filter and $2-$ to $5-\mathrm{MHz}$ convex transducer) (Toshiba, Nemio 35 [SSA-550A], Japan Care Co., Ltd., Japan) after $6 \mathrm{~h}$ of fasting and $20 \mathrm{~min}$ of resting. Color Doppler ultrasonography was used to measure RRI values in the intrarenal segmental arteries. During the examination, spectra were obtained from the level of the arcuate (corticomedullary junction) or interlobar arteries (adjacent to the medullary pyramids) using a 2- to 4-mm Doppler window. All measurements were performed using 


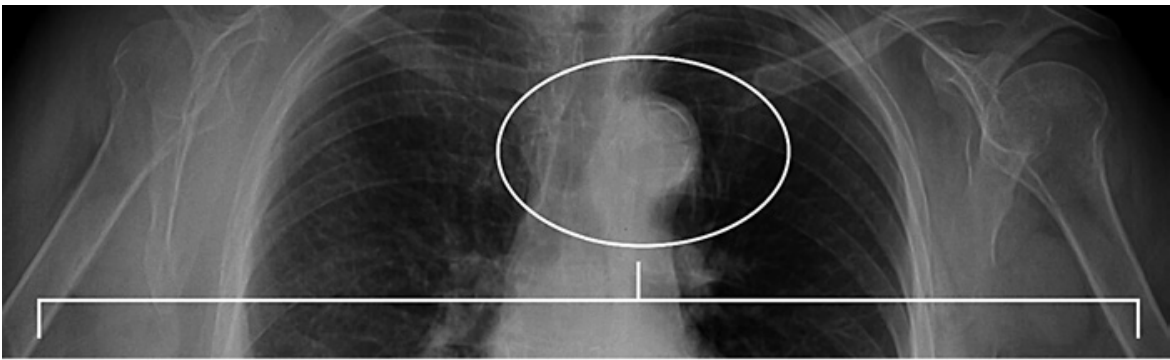

Fig. 1. Grading of aortic arch calcification.
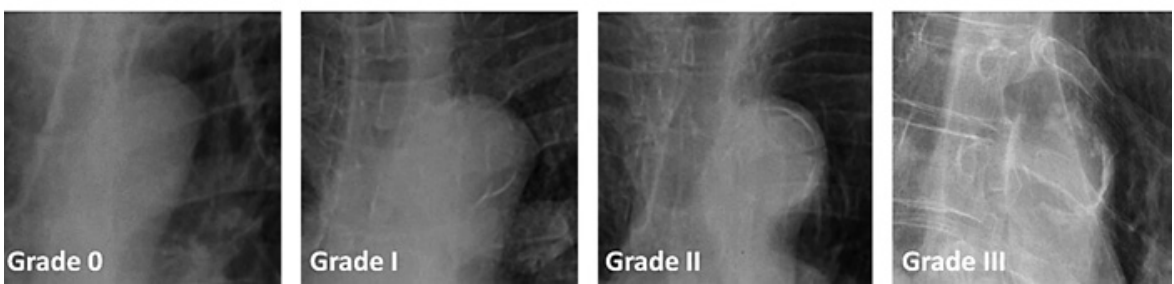

Doppler held at an angle of 30 and $60^{\circ}$ to the vessel lumen. Waveforms were obtained using the lowest pulse repetition frequency that would not generate artifacts. The lowest filter and highest gain controls which would not generate background noise were employed. In the subjects of the present study, the RRI values for the fused part were determined using both the kidneys in the midline. Three RRI values were measured and averaged for both kidneys. The formula used to calculate RRI is as follows: (systolic peak velocity - diastolic peak velocity) / systolic peak velocity [16]. Thirty patients were randomly selected for evaluation of intraobserver variability; the same measurement was repeated after $1 \mathrm{~h}$ in a blinded fashion. The RRI values showed excellent reproducibility with an intraobserver intraclass coefficient of 0.937 and a $95 \%$ confidence interval of $0.872-0.969$.

\section{Laboratory Measurements}

Venous blood samples were obtained from all participants on admission. Creatinine, blood urea nitrogen, glucose, total cholesterol, low-density lipoprotein cholesterol, high-density lipoprotein cholesterol, potassium, sodium, albumin, calcium, phosphate, aspartate aminotransferase, and alanine aminotransferase were quantified using standard techniques. Twenty-four-hour urine albumin concentration was measured by an immunoturbidimetric method. For women, urine collection was repeated if the patient was menstruating. Routine blood chemistry and lipid parameters were measured with a Siemens Advia 1800 analyzer.

\section{Echocardiographic Examination}

All patients were examined using a commercially available system (Vivid 4; GE Medical Systems, Horten, Norway) with a phased-array 3.5-MHz transducer. The conventional M-mode, Bmode, and Doppler parameters were measured according to the guidelines of the American Society of Echocardiography. Left ventricular (LV) end-diastolic and end-systolic diameters as well as posterior and septal wall thickness were measured. LV mass was calculated using the Devereux equation: LV mass $=0.8 \times[1.04$ $(\text { LVEDD + IVST +PWT })^{3}-\left(\right.$ LVEDD $\left.\left.^{3}\right)\right]+0.6$ g, where LVEDD is $\mathrm{LV}$ end-diastolic diameter, IVST is interventricular septal wall thickness, and PWT is posterior wall thickness. LV mass index was

Aortic Arch Calcification and Renal

Resistive Index calculated by dividing LV mass by body surface area. LV hypertrophy was defined as LV mass index $>115 \mathrm{~g} / \mathrm{m}^{2}$ for males and $>95 \mathrm{~g} /$ $\mathrm{m}^{2}$ for females [12].

\section{Statistical Analyses}

The Number Cruncher Statistical System (NCSS) 2007 (Kaysville, UT, USA) program was used for the statistical analysis of the data. Descriptive statistical methods (mean, standard deviation, median, frequency, ratio, minimum, and maximum) were used for the evaluation of study data, where Student's $t$ test was employed for the comparison of quantitative data in two-group comparisons of normally distributed values and Mann-Whitney $U$ test was used for parameters with non-normal distribution. Pearson's and Spearman's correlation analysis were used to evaluate the intervariable relationships. Linear regression analysis was used to examine the risk factors affecting RRI. Pearson's $\chi^{2}$ test was used for the comparison of qualitative data. Significance was considered at a $p$ value $<0.05$.

\section{Results}

The study included 289 patients with hypertension (67.1\% [ $n=194]$ females, $32.9 \%$ [ $n=95]$ males, mean age $63.87 \pm 11.38$ years $)$. Right RRI was found to range from 0.53 to 0.89 , with a mean of $0.71 \pm 0.07$, while left RRI ranged from 0.55 to 0.89 , with a mean of $0.71 \pm 0.07$. RRI was observed to be $<0.70$ in $46.4 \%(n=134)$ of the patients and $\geq 0.70$ in $53.6 \%(n=155)$ of the patients. A statistically significant difference was found between the RRI groups in terms of mean age, with the mean age of the group with RRI $\geq 70$ being higher than that in the group having $\mathrm{RRI}<70(67.71 \pm 10.13$ vs. $59.43 \pm 11.15$, respectively, $p=0.001)$. LV hypertrophy $(p=0.001)$ and acetylsalicylic acid use $(p=0.035)$ were observed to be higher in the group with RRI $\geq 70$ (Table 1 ). AAC was not de- 
Table 1. Demographic characteristics of the study population according to RRI

\begin{tabular}{lccc}
\hline & \multicolumn{2}{l}{ RRI } & $p^{\mathrm{a}}$ \\
\cline { 2 - 3 } & $<70(n=134)$ & $\geq 70(n=155)$ & $0.001^{\mathrm{b}, * *}$ \\
\hline Age, years & $59.43 \pm 11.15$ & $67.71 \pm 10.13$ & $0.792^{\mathrm{a}}$ \\
Female sex & $91(46.9 \%)$ & $103(53.1 \%)$ & $0.733^{\mathrm{b}}$ \\
Body mass index, kg/m ${ }^{2}$ & $30.49 \pm 5.77$ & $30.25 \pm 6.10$ & $0.229^{\mathrm{a}}$ \\
Smoking & $25(39.7 \%)$ & $38(60.3 \%)$ & 0.905 \\
Chronic obstructive lung disease & $10(47.6 \%)$ & $11(52.4 \%)$ & 0.529 \\
Hyperlipidemia & $42(43.8 \%)$ & $54(56.2 \%)$ & 0.276 \\
Statins & $21(39.6 \%)$ & $32(60.4 \%)$ & 0.818 \\
Coronary artery disease & $33(45.2 \%)$ & $40(54.8 \%)$ & 0.171 \\
Cerebrovascular accident & $4(28.6 \%)$ & $10(71.4 \%)$ & 0.119 \\
ACE inhibitors & $91(43.5 \%)$ & $118(56.5 \%)$ & 0.715 \\
Calcium channel blockers & $38(44.7 \%)$ & $37(55.3 \%)$ & 0.801 \\
Beta-blockers & $32(45.1 \%)$ & $39(54.9 \%)$ & $0.035^{*}$ \\
Acetylsalicylic acid & $45(38.8 \%)$ & $51(61.2 \%)$ & $0.001^{* *}$ \\
LV hypertrophy & $24(28.9 \%)$ & $19(41.3 \%)$ & 0.067 \\
Normal geometry & $27(58.7 \%)$ & $56(43.1 \%)$ & $0.001^{* *}$ \\
Concentric remodeling & $74(56.9 \%)$ & $53(66.2 \%)$ & $0.008^{* *}$ \\
Concentric hypertrophy & $27(33.8 \%)$ & $27(81.8 \%)$ & $0.001^{* *}$ \\
Eccentric hypertrophy & $6(18.2 \%)$ & & \\
\hline
\end{tabular}

Values are presented as mean \pm standard deviation or $n(\%)$. ACE, angiotensin-converting enzyme; LV, left

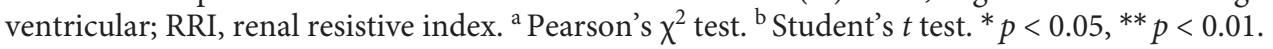

Table 2. Association between RRI and AAC

\begin{tabular}{lrrl}
\hline \multirow{2}{*}{ AAC } & \multicolumn{2}{l}{ RRI } & \multirow{2}{*}{$p^{\mathrm{a}}$} \\
\cline { 2 - 3 } & $<70$ & \multicolumn{1}{l}{$\geq 0$} \\
\hline Absence & $74(85.1 \%)$ & $13(14.9 \%)$ & $0.001^{* *}$ \\
Presence & $60(29.7 \%)$ & $142(70.3 \%)$ & \\
\hline Absence & $74(85.1 \%)$ & $13(14.9 \%)$ & $0.001^{* *}$ \\
Grade I & $42(38.2 \%)$ & $68(61.8 \%)$ & \\
Grade II & $10(14.3 \%)$ & $60(85.7 \%)$ & \\
Grade III & $8(36.4 \%)$ & $14(63.6 \%)$ & \multirow{2}{*}{$0.001^{* *}$} \\
\hline <grade II & $116(58.9 \%)$ & $81(41.1 \%)$ & \\
$\geq$ grade II & $18(19.6 \%)$ & $74(80.4 \%)$ & \\
\hline
\end{tabular}

Values are presented as $n(\%)$. AAC, aortic arch calcification; RRI, renal resistive index. ${ }^{\text {a }}$ Pearson's $\chi^{2}$ test. ${ }^{* *} p<0.01$.

tected in $30.1 \%(n=87)$ of the subjects, while it was detected in $69.9 \%(n=202)$ of the patients as grade $\mathrm{I}$ in $38.1 \%(n=110)$, grade II in $24.2 \%(n=70)$, and grade III in $7.6 \%(n=22)$ of the patients. A statistically significant association was found between RRI and the presence and grade of AAC $(p=0.001)$ (Table 2$)$.
A total of 156 out of the 289 patients (54.0\%) had CKD. Patients were divided into two groups according to the presence of CKD. Patients with CKD were older (65.22 \pm 11.96 vs. $62.29 \pm 10.46$ years; $p=0.028)$. The prevalence of smoking (41 [65.1\%] vs. 22 [34.9\%]; $p=0.046)$ and LV hypertrophy (53 [63.9\%] vs. $30[36.1 \%] ; p=0.032$ ) was found to be higher in patients with CKD. In addition, patients with CKD were found to have higher RRIs (0.71 [0.7] vs. 0.70.0 [0.6]; $p=0.045)$ and AAC prevalence (118 [58.4\%] vs. $84[41.6 \%] ; p=0.021$.

RRI showed a negative correlation between GFR ( $r=$ $-0.267 ; p<0.01)$, serum albumin level $(r=-0.189$; $p<$ $0.01)$, body surface area $(r=-0.120 ; p<0.05)$, and LV ejection fraction $(r=-0.188 ; p<0.01)$, which was statistically significant. On the other hand, a positive correlation was found between urea $(r=0.242 ; p<0.01)$, serum potassium level $(r=0.140 ; p<0.05)$, albuminuria $(r=0.187 ; p<0.05)$, LV end-diastolic diameter $(r=0.207$; $p<0.01)$, LV endsystolic diameter $(r=0.227 ; p<0.01)$, left atrium diameter $(r=0.126 ; p<0.05)$, interventricular septal thickness $(r=$ 0.215 ; $p<0.01)$, posterior wall thickness $(r=0.136$; $p<$ $0.05)$, LV mass index $(r=0.320 ; p<0.01)$, pulse pressure $(r=0.211 ; p<0.05)$, and systolic blood pressure $(r=0.146$; $p<0.05$ ), which was statistically significant (Table 3 ). 
Table 3. Relationship between RRI and laboratory findings, echocardiographic, and blood pressure measurements

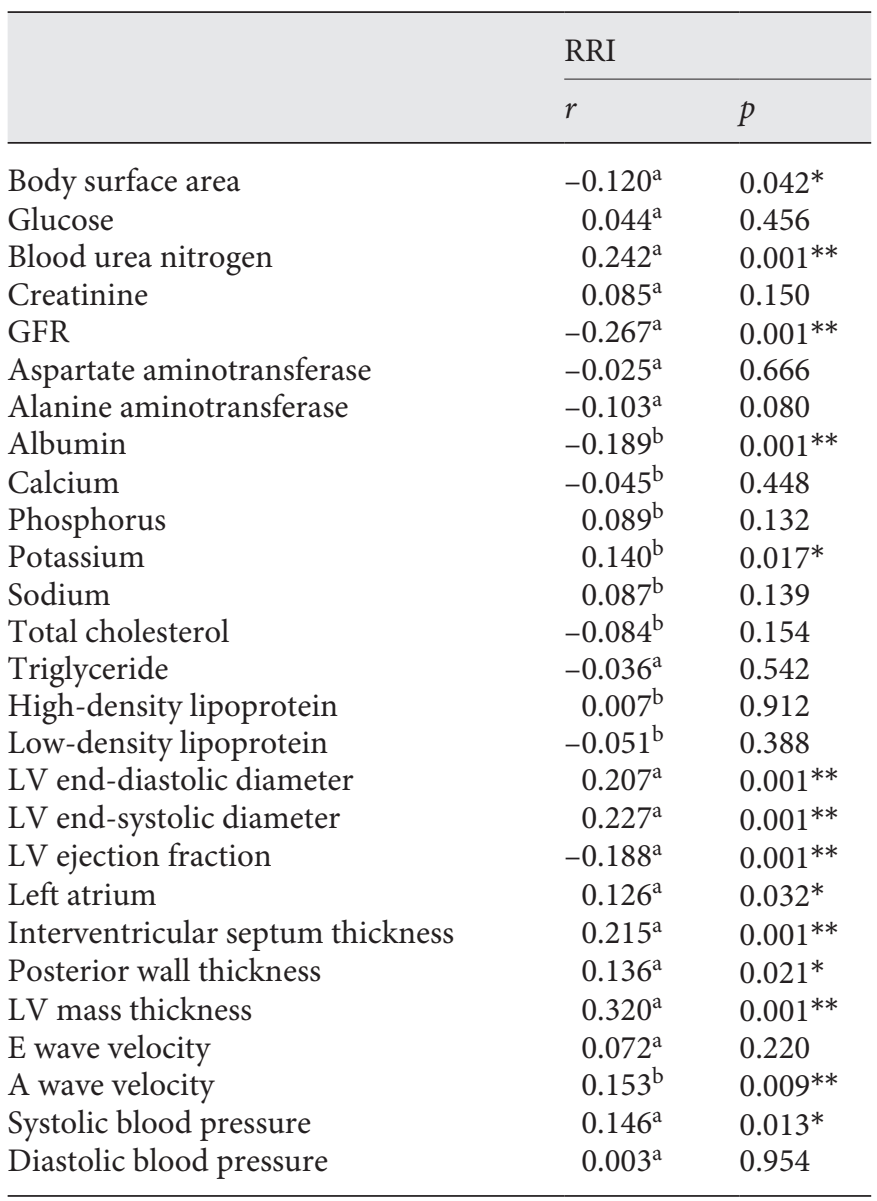

GFR, glomerular filtration rate; LV, left ventricular; RRI, renal resistive index. ${ }^{\text {a }}$ Pearson's correlation coefficient. ${ }^{\text {b }}$ Spearman's correlation coefficient. ${ }^{*} p<0.05,{ }^{* *} p<0.01$.

\section{Linear Regression Analysis}

Presence of AAC, pulse pressure, albuminuria, age, GFR, serum albumin level, LV ejection fraction, left atrium diameter, serum potassium level, A velocity, LV mass index, body surface area, normal geometry, concentric hypertrophy, eccentric hypertrophy, and acetylsalicylic acid use were analyzed by linear regression analysis.

A multiple linear regression analysis was carried out to test whether presence of AAC significantly predicted RRI. The results of the regression indicated that the model explained $39.2 \%$ of the variance and that the model was a significant predictor of RRI $\left(F_{(16,72)}=10.964 ; p<0.001\right)$. It was found that presence of AAC significantly predicted RRI $(\beta=0.053 ; p<0.001)$ (Table 4$)$.

Aortic Arch Calcification and Renal

Resistive Index

\section{Discussion}

This study establishes a strong and independent relationship between AAC, as evaluated by chest X-ray, and RRI, an indicator of the intrarenal hemodynamic parameter.

Hypertension causes remodeling, endothelial dysfunction, fibrosis, and calcification, leading to significant vascular changes. Vascular calcification is one of the most important indicators of hypertension-related vascular damage. Inflammation, oxidative stress, advanced age, and renin-angiotensin system are important factors involved in the development of vascular calcification [1719]. Angiotensin 2, the substrate of the renin-angiotensin system, plays an active role in the growth and differentiation of vascular smooth muscle cells [18]. Vascular smooth muscle cells are composed of mesenchymal cells such as osteoblasts. Influenced by various environmental factors, vascular smooth muscle cells get converted into an osteoblast phenotype and produce calcium. Thus, vascular calcification begins with the production of calcium in the intima or media [20]. AAC is the prototype of vascular calcification. The most significant pathophysiological justification for the relationship between AAC and RRI is that the formation of AAC results in decreased vascular compliance and increased arterial stiffness [8]. Increased arterial stiffness reduces compliance. Arterial compliance is a major characteristic of the pulsatile component of blood pressure. The elastic wall of the aorta stretches during systole and recoils during diastole. Thus, peripheral blood flow, especially renal blood flow, is ensured. Increased aortic stiffness decreases aortic compliance, leading to decreased renal blood flow [21]. A decrease in the renal blood flow leads to an increase in RRI.

Vascular calcification is a systemic entity that effects the whole vascular bed. In another study, a strong and independent correlation was found between coronary artery calcification and AAC [22]. In addition, a strong correlation was reported between AAC and renal artery calcification, which is an important indicator of renal artery disease [23]. Renal artery calcification has significant effects on renal hemodynamic parameters. According to the authors, another pathophysiological explanation for the correlation between AAC and RRI is that systemic vascular calcification, especially renal artery calcification, impairs renal blood flow, thus increasing RRI. The present study demonstrated an association between AAC and RRI, thus offering more robust evidence for the association between vascular calcification and RRI [24]. The present study also established a correlation between age

Med Princ Pract 2019;28:133-140 137 
Table 4. Linear regression analysis for RRI

\begin{tabular}{lcccc}
\hline Factor & Unstandardized $\beta$ & SE & Standardized $\beta$ & $p$ value \\
\hline Constant & 0.443 & 0.102 & & $<0.001$ \\
AAC presence & 0.053 & 0.009 & 0.356 & $<0.001$ \\
Pulse pressure & 0.001 & 0.00 & 0.174 & $<0.001$ \\
Albuminuria & 5.8 & 0.000 & 0.176 & $<0.001$ \\
Age & 0.001 & 0.000 & 0.204 & 0.009 \\
Glomerular filtration rate & 0.000 & 0.000 & 0.121 & 0.063 \\
Serum albumin level & -0.008 & 0.011 & -0.036 & 0.475 \\
LVEF & 0.000 & 0.000 & -0.025 & 0.640 \\
Left atrium & 0.000 & 0.001 & 0.018 & 0.745 \\
Potassium & 0.011 & 0.008 & 0.067 & 0.178 \\
A velocity & 0.000 & 0.000 & 0.74 & 0.132 \\
LVMI & 0.000 & 0.000 & 0.067 & 0.402 \\
Body surface area & -0.010 & 0.188 & -0.003 & 0.956 \\
Normal geometry & 0.010 & 0.010 & 0.051 & 0.327 \\
Concentric hypertrophy & 0.011 & 0.011 & 0.072 & 0.312 \\
Eccentric hypertrophy & 0.018 & 0.013 & 0.085 & 0.174 \\
Acetylsalicylic acid use & 0.004 & 0.007 & 0.026 & 0.604 \\
\hline
\end{tabular}

Model fit: $R=0.626, R^{2}=0.392$, adjusted $R^{2}=0.359$. AAC, aortic arch calcification; LVEF, left ventricular ejection fraction; LVMI, left ventricular mass index; RRI, renal resistive index; SE, standard error.

and increase in RRI. Several reports indicate that a correlation exists between RRI and age. The reason for an increase in RRI with age was suggested to be increased arterial stiffness and decreased vascular compliance with age [25]. Also, a strong reverse correlation between GFR and RRI was found in this study. A few studies showing a correlation between RRI and GFR have been reported in the literature, which are in support of the results of the present study [9]. In the present study, a positive correlation was found between LV dilatation, functions, and RRI. Ratto et al. [26] also reported a correlation between LV dilatation, functions, and RRI. LV hypertrophy (eccentric and concentric hypertrophy) has been established to be an independent predictor of RRI in the present study. Increase in arterial stiffness and blood pressure load may increase RRI and result in the development of LV hypertrophy. Alterini et al. [27] demonstrated a correlation of LV mass index and LV hypertrophy with RRI in hypertensive patients, which is in accordance with the present study.

RRI is the result of an interaction between renal hemodynamic parameters and systemic hemodynamic factors. RRI has been reported to be associated with many renal diseases as well as with important cardiovascular diseases [28]. RRI, which is closely associated with LV systolic and diastolic function, is indicative of progression of renal disease as well as progression of heart failure [28]. Simi- larly, it is known that RRI is closely related to aortic stiffness and atherosclerosis [29]. Pulse pressure is formed by the interaction of cardiac functions with the vascular structures. A strong association between RRI and pulse pressure has been reported [30]. In concordance with this, in the present study we found a significant association between RRI, pulse pressure, and albuminuria. However, we were not able show a significant association between RRI, E/A, and LV ejection fraction.

Cardiorenal syndrome is an example of the interlinking of cardiac and renal diseases. Cardiovascular morbidity and mortality are increased in this syndrome [31]. The present study also found a correlation between LV dilatation, functions, and RRI, thus supporting the possibility of cardiorenal syndrome. In addition, aortic calcification was found to be an independent and strong predictor of RRI. Also, LV function and hypertrophy are known to be correlated with aortic calcification [32]. It can be speculated that vascular calcification may be involved in the pathogenesis of cardiorenal syndrome, as evaluated by the results of the present study [33]. In addition, it is clear that aortic calcification may be a part of cardiorenal syndrome.

\section{Limitations}

The limitations of this study include the small sample size, the study being a single-center study, no demonstra- 
tion of the pathophysiological relationship between RRI and $\mathrm{AAC}$, and no investigation of end-organ damage resulting from hypertension.

\section{Conclusion}

An independent and a strong correlation between AAC and RRI, an indicator of renal hemodynamic parameters, has been established to occur in patients with hypertension. AAC, evaluated by chest X-ray and a commonly used and readily available procedure in clinical practice, may be an indicator of increased RRI.

\section{Statement of Ethics}

The study was planned according to the recommendations set forth by the Declaration of Helsinki on Biomedical Research Involving Human Subjects. The Institutional Ethics Committee approved the study protocol; informed consent was taken from each participant.

\section{Disclosure Statement}

The authors have no conflicts on interest to declare.

\section{References}

1 Verdecchia P, Clement D, Fagard R, Palatini P, Parati G. Blood Pressure Monitoring. Task force III: target-organ damage, morbidity and mortality. Blood Press Monit. 1999 Dec;4(6): 303-17.

2 Nakayama M, Sato T, Miyazaki M, Matsushima M, Sato H, Taguma Y, et al. Increased risk of cardiovascular events and mortality among non-diabetic chronic kidney disease patients with hypertensive nephropathy: the Gonryo study. Hypertens Res. 2011 Oct;34(10):110610.

3 Ikee R, Kobayashi S, Hemmi N, Imakiire T, Kikuchi Y, Moriya H, et al. Correlation between the resistive index by Doppler ultrasound and kidney function and histology. Am J Kidney Dis. 2005 Oct;46(4):603-9.

4 Cauwenberghs N, Kuznetsova T. Determinants and Prognostic Significance of the Renal Resistive Index. Pulse (Basel). 2016 Apr; 3(3-4):172-8.

5 Mancia G, De Backer G, Dominiczak A, Cifkova R, Fagard R, Germano G, et al.; ESHESC Task Force on the Management of Arterial Hypertension. 2007 ESH-ESC Practice Guidelines for the Management of Arterial Hypertension: ESH-ESC Task Force on the Management of Arterial Hypertension. J Hypertens. 2007 Sep;25(9):1751-62.

6 O'Rourke C, Shelton G, Hutcheson JD, Burke MF, Martyn T, Thayer TE, et al. Calcification of Vascular Smooth Muscle Cells and Imaging of Aortic Calcification and Inflammation. J Vis Exp. 2016 May;(111). https://doi. org/10.3791/54017.

7 Tsao CW, Pencina KM, Massaro JM, Benjamin EJ, Levy D, Vasan RS, et al. Cross-sectional relations of arterial stiffness, pressure pulsatility, wave reflection, and arterial calcification. Arterioscler Thromb Vasc Biol. 2014 Nov;34(11):2495-500.

8 Mackey RH, Venkitachalam L, Sutton-Tyrrell K. Calcifications, arterial stiffness and atherosclerosis. Adv Cardiol. 2007;44:234-44.
9 Calabia J, Torguet P, Garcia I, Martin N, Mate G, Marin A, et al. The relationship between renal resistive index, arterial stiffness, and atherosclerotic burden: the link between macrocirculation and microcirculation. J Clin Hypertens (Greenwich). 2014 Mar; 16(3): $186-91$.

10 Berni A, Ciani E, Bernetti M, Cecioni I, Berardino S, Poggesi L, et al. Renal resistive index and low-grade inflammation in patients with essential hypertension. J Hum Hypertens. 2012 Dec;26(12):723-30.

11 National Cholesterol Education Program (NCEP) Expert Panel on Detection, Evaluation, and Treatment of High Blood Cholesterol in Adults (Adult Treatment Panel III). Third Report of the National Cholesterol Education Program (NCEP) Expert Panel on Detection, Evaluation, and Treatment of High Blood Cholesterol in Adults (Adult Treatment Panel III) final report. Circulation. 2002 Dec;106(25):3143-421.

12 National Kidney Foundation. K/DOQI clinical practice guidelines for chronic kidney disease: evaluation, classification, and stratification. Am J Kidney Dis. 2002 Feb;39(2 Suppl 1):S1-266.

13 Levey AS, Stevens LA, Schmid CH, Zhang YL, Castro AF 3rd, Feldman HI, et al.; CKD-EPI (Chronic Kidney Disease Epidemiology Collaboration). A new equation to estimate glomerular filtration rate. Ann Intern Med. 2009 May;150(9):604-12.

14 Obesity: preventing and managing the global epidemic. Report of a WHO consultation. World Health Organ Tech Rep Ser. 2000; 894:i-xii.

15 Mosteller RD. Simplified calculation of bodysurface area. N Engl J Med. 1987 Oct;317(17): 1098.

16 Tublin ME, Bude RO, Platt JF. Review. The resistive index in renal Doppler sonography: where do we stand? AJR Am J Roentgenol. 2003 Apr;180(4):885-92.
17 Aikawa E, Nahrendorf M, Figueiredo JL, Swirski FK, Shtatland T, Kohler RH, et al. Osteogenesis associates with inflammation in early-stage atherosclerosis evaluated by molecular imaging in vivo. Circulation. 2007 Dec;116(24):2841-50.

18 Savoia C, Burger D, Nishigaki N, Montezano A, Touyz RM. Angiotensin II and the vascular phenotype in hypertension. Expert Rev Mol Med. 2011 Mar; 13:e11.

19 You H, Yang H, Zhu Q, Li M, Xue J, Gu Y, et al. Advanced oxidation protein products induce vascular calcification by promoting osteoblastic trans-differentiation of smooth muscle cells via oxidative stress and ERK pathway. Ren Fail. 2009;31(4):313-9.

20 Chen NX, Moe SM. Vascular calcification: pathophysiology and risk factors. Curr Hypertens Rep. 2012 Jun;14(3):228-37.

21 Ratto E, Leoncini G, Viazzi F, Vaccaro V, Falqui V, Parodi A, et al. Ambulatory arterial stiffness index and renal abnormalities in primary hypertension. J Hypertens. 2006 Oct; 24(10):2033-8.

22 Adar A, Erkan H, Gokdeniz T, Karadeniz A, Cavusoglu IG, Onalan O. Aortic arch calcification is strongly associated with coronary artery calcification. Vasa. 2015 Mar;44(2):10614.

23 Allison MA, DiTomasso D, Criqui $\mathrm{MH}$, Langer RD, Wright CM. Renal artery calcium: relationship to systemic calcified atherosclerosis. Vasc Med. 2006 Nov; 11(4):232-8.

24 Stefan G, Capusa C, Stancu S, Petrescu L, Nedelcu ED, Andreiana I, et al. Abdominal aortic calcification and renal resistive index in patients with chronic kidney disease: is there a connection? J Nephrol. 2014 Apr;27(2): 173-9. 
25 Kaiser C, Götzberger M, Landauer N, Dieterle C, Heldwein W, Schiemann U. Age dependency of intrarenal resistance index (RI) in healthy adults and patients with fatty liver disease. Eur J Med Res. 2007 May; 12(5):1915.

26 Ratto E, Viazzi F, Bonino B, Gonnella A, Garneri D, Parodi EL, et al. Left ventricular dilatation and subclinical renal damage in primary hypertension. J Hypertens. 2015 Mar;33(3): 605-11; discussion 611.

27 Alterini B, Mori F, Terzani E, Raineri M, Zuppiroli A, De Saint Pierre G, et al. Renal resistive index and left ventricular hypertrophy in essential hypertension: a close link. Ann Ital Med Int. 1996 Apr-Jun;11(2):107-13.
28 Di Nicolò P, Granata A. Renal Resistive Index: not only kidney. Clin Exp Nephrol. 2017 Jun;21(3):359-66.

29 Hashimoto J, Ito S. Central pulse pressure and aortic stiffness determine renal hemodynamics: pathophysiological implication for microalbuminuria in hypertension. Hypertension. 2011 Nov;58(5):839-46.

30 Lee MK, Hsu PC, Chu CY, Lee WH, Chen SC, Chen HC, et al. Significant Correlation between Brachial Pulse Pressure Index and Renal Resistive Index. Acta Cardiol Sin. 2015 Mar;31(2):98-105.
31 Palazzuoli A, Ronco C. Cardio-renal syndrome: an entity cardiologists and nephrologists should be dealing with collegially. Heart Fail Rev. 2011 Nov; 16(6):503-8.

32 Cho IJ, Chang HJ, Park HB, Heo R, Shin S, Shim CY, et al. Aortic calcification is associated with arterial stiffening, left ventricular hypertrophy, and diastolic dysfunction in elderly male patients with hypertension. J Hypertens. 2015 Aug;33(8):1633-41.

33 Guo J, Lu L, Hua Y, Huang K, Wang I, Huang $\mathrm{L}$, et al. Vasculopathy in the setting of cardiorenal syndrome: roles of protein-bound uremic toxins. Am J Physiol Heart Circ Physiol. 2017 Jul;313(1):H1-13. 